\title{
Integrated Structure and Control Design of Modular Tensegrities
}

\author{
Milenko Masic, Robert E. Skelton, Mauricio Carvalho de Oliveira \\ Mechanical and Aerospace Engineering Department, Structural Systems \& Control Lab, \\ University of California San Diego, 9500 Gilman Drive, La Jolla, CA 92093-0411, USA
}

\begin{abstract}
This paper demonstrates a method for prestress optimization of tensegrity structures resulting in their optimal mixed dynamic and control performance. Prestress of a structure is parameterized using force-density variables that appear linearly in its linearized dynamic model. A feasible region for these parameters is defined in terms of the extreme directions of the prestress cone. A numerical method is proposed for computing this particular basis for the structure prestress cone. The problem is solved using a gradient method based on the sensitivity analysis. A numerical example of a cantilevered planar tensegrity beam is shown.
\end{abstract}

Keywords: Tensegrity Structures, Control, Optimization, Prestress

\section{INTRODUCTION}

Tensegrity structure are prestressable, stable, truss-like system involving string elements. They were originally introduced and used as artistic forms. The renewed interest in their engineering applications have been generated by their favorable properties that include reconfigurability and structural efficiency. Because of the presence of the controllable string elements, tensegrities are suitable for applications in shape morphing structures capable of undergoing large shape changes. At the same time, utilization of the materials specialized for tensile loads, that have superior mechanical properties over materials available for compressive elements, yields lightweight structures. Related engineering problems have been topics of recent studies.

A majority of the published tensegrity research concerns form-finding and rigidity problems, [1], [2], [3], [4], [5]. The methods for optimal structural design of tensegrity structures have been proposed recently, [6], [7]. Several different dynamic models of tensegrities have been derived, e.g., [8], [9], and a number of control strategies are available, e.g., [10], [11], [12], [13], [14], [15], [16].

Traditionally, the structure and control designs have been two independent engineering problems. Suitability of structures for latter control implementation is usually ignored during their design phase which yields structures with severe performance limitations. Although designing structures and their control jointly has a clear advantage, mathematical tools for solving this problem are of a limited scope. There has been a number of results available on this subject, e.g. [17], [18], [19], [20], [21], [22]. Because of the non-convex nature of the problem the available methods for joint structure and control optimization provide at most a convergence to a local minimum.
In this paper, we seek the distribution of the structure prestress that yields the optimal LQR performance of the system for a given nodal configuration, support conditions and load scenario. We use the fact that the prestress parameters appear linearly in the linearized dynamic model to analytically define problem gradients using sensitivity analysis.

The paper is outlined as follows. We define tensegrity equilibrium conditions and other preliminary notions in Section 2. The linearized dynamic model is presented in Section 3 along with the definition of the feasible set of the design parameters. In Section 4, the optimization problem is defined first. This section is concluded with a numerical example. Conclusions are given in Section 5.

\section{PRELIMINARY NOTIONS-TENSEGRITY EQUILIBRIUM CONDITIONS AND CONSTITUTIVE EQUATIONS}

Let the set $\mathbb{N}$ of $n_{n}$ nodes of a tensegrity structure be given, and let the node $\nu_{j} \in \mathbb{N}$ be located at the position defined by the nodal vector $\mathbf{p}_{j} \in \mathbb{R}^{3}$. Denote the set of all nodal vectors $\mathbb{P}$.

Let the set $\mathbb{E}$ of $n_{e}$ elements of the structure be given, and let $\mathbb{E}_{s}$ and $\mathbb{E}_{b}$ denote its partitions into the sets of $n_{s}$ strings and $n_{b}$ bars respectively. The scalar $z_{i}$ in the definition of the element $e_{i}=\left\{\left[\nu_{j}, \nu_{k}\right], z_{i}\right\} \in \mathbb{E}$ that connects the nodes $\nu_{j}$ and $\nu_{k}$ of the tensegrity, identifies the type of the element so that,

$$
z_{i}=\left\{\begin{aligned}
1, & e_{i} \in \mathbb{E}_{s}, \\
-1, & e_{i} \in \mathbb{E}_{b} .
\end{aligned}\right.
$$

We define an element vector, $\mathbf{g}_{i} \in \mathbb{R}^{3}$, as a vector along the length of an element $e_{i}=\left\{\left[\nu_{k}, \nu_{j}\right], z_{i}\right\}$. It emanates from the first node $\nu_{k}$ and terminates at the second node $\nu_{j}$ of the element, i.e.,

$$
\mathbf{g}_{i}=\mathbf{p}_{j}-\mathbf{p}_{k} .
$$

It is obvious that magnitude of an element vector $\mathbf{g}_{i}$ is equal to its length $\left\|\mathbf{g}_{i}\right\|$, which is denoted by $l_{i}$.

We define now the element force vector $\mathbf{f}_{j i} \in \mathbb{R}^{3}$ that represents the contribution of the internal force of the element $e_{i}$, to the total nodal force at the node $\nu_{j}$ as,

$$
\mathbf{f}_{j i}=c_{j i}\left(\lambda_{i}+\lambda_{i}^{d}\right) \mathbf{g}_{i}, \quad f_{i}=\lambda_{i}\left\|\mathbf{g}_{i}\right\|=\lambda_{i} l_{i},
$$

where the element elastic force density $\lambda_{i}$ and the damping force density $\lambda_{i}^{d}$ are scalars. Obviously scalars $c_{j i}$ in (2) that are defined as typical elements of the matrix $C(\mathbb{E}) \in$ $\mathbb{R}^{n_{n} \times n_{e}}$, have one of the three possible values, $c_{j i}= \pm 1$ or $c_{j i}=0$. 
Let the vector space of vectors $\mathbf{x} \in \mathbb{R}_{m}^{n}$ be defined as follows,

$$
\mathbf{x}^{T}=\left[\begin{array}{lll}
\mathbf{x}_{1}^{T} & \ldots & \mathbf{x}_{n}^{T}
\end{array}\right], \quad \mathbf{x}_{i} \in \mathbb{R}^{m}, \quad \mathbb{R}^{m}=\mathbb{R}_{1}^{m} .
$$

Vector of nodal vectors $\mathbf{p} \in \mathbb{R}_{3}^{n_{n}}$, vector of element vectors $\mathrm{g}(\mathbb{E}, \mathbb{P}) \in \mathbb{R}_{3}^{n_{e}}$, vector of force densities $\boldsymbol{\lambda} \in \mathbb{R}^{n_{e}}$ and vector $\mathbf{z} \in \mathbb{R}^{n_{e}}$ are formed by collecting all node vectors $\mathbf{p}_{i}$, element vectors $\mathbf{g}_{i}$, force densities $\lambda_{i}$ and all individual element type identifiers.

Let the member-node incidence matrix of the oriented graph associated with $\mathbb{E}$ be denoted $M(\mathbb{E}) \in \mathbb{R}^{n_{e} \times n_{n}}$ and let $\mathbf{M} \in \mathbb{R}^{3 n_{e} \times 3 n_{n}}$ be defined as $\mathbf{M}=M \otimes I_{3}$. The typical element $m_{i j}$ of the matrix $M$ is $m_{i j}=1$ or $m_{i j}=-1$ if the element $e_{i}$ terminates at or emanates from the node $\nu_{j}$, otherwise $m_{i j}=0$. Let the $n_{s}$ string elements in $\mathbb{E}_{s}$ be numbered first. Then, vector $\mathbf{g}$ and matrix $\mathbf{M}$ can be partitioned as follows,

$\mathbf{g}=\left[\begin{array}{l}\mathbf{g}_{s} \\ \mathbf{g}_{b}\end{array}\right]=\mathbf{M p}, \quad \mathbf{M}=\left[\begin{array}{l}\mathbf{S}^{T} \\ \mathbf{B}^{T}\end{array}\right], \quad \mathbf{S} \in \mathbb{R}^{3 n_{n} \times 3 n_{s}}$.

One can show that equilibrium conditions for the prestressed structure with properly loaded strings, and matrix $\mathbf{C}=C \otimes I_{3}$ can be defined and written as,

$$
\begin{gathered}
\mathbf{C} \tilde{\mathbf{g}} \boldsymbol{\lambda}=0, \quad\|\boldsymbol{\lambda}\|>0, \quad \underline{\lambda}_{i} \geq 0, \quad e_{i} \in \mathbb{E}_{s}, \\
\mathbf{C}=\left[\begin{array}{ll}
-\mathbf{S} & \mathbf{B}
\end{array}\right], \quad \mathbf{g}=\mathbf{M} \mathbf{p}=\left[\begin{array}{c}
\mathbf{S}^{T} \\
\mathbf{B}^{T}
\end{array}\right] \mathbf{p},
\end{gathered}
$$

if the linear operator $\sim$ acting on the vector $\mathbf{x} \in \mathbb{R}_{m}^{n}$ is defined as follows,

$$
\tilde{\mathbf{x}}:=\operatorname{blockdiag}\left\{\mathbf{x}_{1} \ldots, \mathbf{x}_{i}, \ldots \mathbf{x}_{n}\right\} \in \mathbb{R}^{m n \times n}, \quad \mathbf{x}_{i} \in \mathbb{R}^{m}
$$

The relationship between the elastic force density variables and the structural parameters depends on the particular constitutive model. Let the elements of the structure be built from a linear elastic material, and let their mass, crosssectional area, Young's modulus, rest lengths, and viscous damping coefficients be given in the following vectors, $\mathbf{m}, \mathbf{a}, \mathbf{y}, \mathbf{l}_{0}, \boldsymbol{\mu} \in \mathbb{R}^{n_{e}}$. Elastic force densities can then be computed from the Hooke's law,

$$
\lambda_{i}(\mathbf{p})=\left\{\begin{array}{l}
z_{i} \frac{y_{i} a_{i}}{l_{i}(\mathbf{p}) l_{0_{i}}}\left(l_{i}(\mathbf{p})-l_{0_{i}}\right), \\
0, \quad \text { if } e_{i} \in \mathbb{E}_{s} \text { and } l_{i}(\mathbf{p}) \leq l_{0_{i}} .
\end{array}\right.
$$

Similarly, damping force densities can be computed as,

$$
\lambda_{i}^{d}(\mathbf{p}, \dot{\mathbf{p}})=\left\{\begin{array}{l}
z_{i} \frac{1}{l_{i}(\mathbf{p})} \mu_{i} l_{i}(\mathbf{p}, \dot{\mathbf{p}}), \\
0, \quad e_{i} \in \mathbb{E}_{s} \text { and } l_{i}(\mathbf{p}) \leq l_{0_{i}} .
\end{array}\right.
$$

The non-smooth nonlinear relationships in (6) and (7) reflect the fact that the string elements cannot transmit a load when they are slack.

A more detailed explanation of tensegrity equilibrium equations (4-5) is given in [5].

\section{A LUMPED MASS DYNAMIC MODEL OF A TENSEGRITY STRUCTURE}

Assume that all string elements of a stationary tensegrity structure are taut when the structure is subjected to the prestress $\boldsymbol{\lambda}$ in the equilibrium configuration $\mathbf{p}$. The finite variations of the equilibrium variables $\mathbf{p}$ and $\boldsymbol{\lambda}$ are denoted $\mathbf{u}$ and $\Delta \boldsymbol{\lambda}$ respectively. Assume further that the set of independent nodal constraints are defined in the following form,

$$
C_{u} \mathbf{u}=0
$$

and that the SVD of the matrix $C_{u}$ is,

$$
\begin{gathered}
C_{u}=U \Sigma V^{T}=U\left[\begin{array}{ll}
\Sigma_{1} & 0
\end{array}\right]\left[\begin{array}{l}
V_{1}^{T} \\
V_{2}^{T}
\end{array}\right]=U \Sigma_{1} V_{1}^{T}, \\
U U^{T}=U^{T} U=I, \quad V V^{T}=V^{T} V=I .
\end{gathered}
$$

Hence, $\mathbf{u}=V_{2} \underline{\mathbf{u}}$, and the linearized lumped mass model of the structure in the presence of the external nodal forces, given in the vector $\mathbf{w} \in \mathbb{R}_{3}^{n_{n}}$, can be defined as,

$$
\begin{aligned}
& V_{2}^{T} \mathcal{M} V_{2} \underline{\ddot{\mathbf{u}}}+V_{2}^{T} \mathcal{D} V_{2} \underline{\dot{\mathbf{u}}}+V_{2}^{T} \mathcal{K}(\boldsymbol{\lambda}, \mathbf{p}, \mathbf{y}, \mathbf{a}) V_{2} \underline{\mathbf{u}}= \\
& =V_{2}^{T} \mathbf{C} \tilde{\mathbf{g}} \Delta \boldsymbol{\lambda}+V_{2}^{T} \mathbf{w} .
\end{aligned}
$$

For more details on the derivation of this model of constrained structure dynamics consult [23]. Here, we give the final compact form of the mass, damping and stiffness matrices, $\mathcal{M}, \mathcal{D}$ and $\mathcal{K}$,

$$
\begin{aligned}
& \mathcal{K}(\boldsymbol{\lambda}, \mathbf{p}, \mathbf{y}, \mathbf{a})=\mathbf{C} \tilde{\mathbf{g}} \tilde{\mathbf{y}} \tilde{\mathbf{a}} \tilde{\mathbf{l}}^{-3} \widetilde{\mathbf{g}}^{T} \mathbf{C}^{T}-\mathbf{C} \hat{\boldsymbol{\lambda}} \mathbf{M} \\
& \mathcal{D}=\mathbf{C} \tilde{\mathbf{g}} \tilde{\mathbf{l}}^{-2} \tilde{\boldsymbol{\mu}} \widetilde{\mathbf{g}}^{T} \mathbf{C}^{T}, \\
& \mathcal{M}=\hat{\mathbf{m}}^{n}, \quad \mathbf{m}^{n}=0.5 \operatorname{abs}(C) \mathbf{m},
\end{aligned}
$$

\section{A. State space model of the system}

A parameterized state space form of the linear dynamic model of the structure is obtained after identifying, nodal displacements $\underline{\mathbf{u}}$ and nodal velocities $\underline{\dot{\mathbf{u}}}$ as the state variables, $\Delta \boldsymbol{\lambda}$ as the control input variables, and $\mathrm{w}$ as the exogenous inputs,

$$
\begin{gathered}
\dot{x}=A(\boldsymbol{\lambda}) x+B \Delta \boldsymbol{\lambda}+B_{w} w \\
0 \\
A=\left[\begin{array}{cc}
I \\
-\underline{\mathcal{M}}^{-1} \underline{\mathcal{K}}(\boldsymbol{\lambda}, \mathbf{p}, \mathbf{y}, \mathbf{a}) & -\underline{\mathcal{M}}^{-1} \mathcal{D}
\end{array}\right], \\
0 \\
B=\left[\begin{array}{c}
0 \\
\underline{\mathcal{M}}^{-1} V_{2}^{T} \mathbf{C} \tilde{\mathbf{g}}
\end{array}\right], B_{w}=\left[\begin{array}{c}
\underline{\mathcal{M}}^{-1} V_{2}^{T}
\end{array}\right] .
\end{gathered}
$$

1) Identifying the structure design freedom: Recall that the prestress parameters, $\boldsymbol{\lambda}$, of the structure model in (9) represent equilibrium force densities. Any freedom in the model must comply with this constraint. Consider an equilibrium tensegrity structure that in the configuration $\mathbf{p}$ has a multidimensional prestress cone with the basis $\Lambda(\mathbf{p})$, and let,

$$
\lambda=\Lambda(\mathbf{p}) \underline{\underline{\lambda}} .
$$

Let the columns of the prestress cone basis, $\Lambda(\mathbf{p})$, represent the extreme directions of the tensegrity cone, so that every feasible force density vector, $\boldsymbol{\lambda} \in \Lambda(\mathbf{p})$, can be written as, $\boldsymbol{\lambda}=\Lambda(\mathbf{p}) \underline{\underline{\boldsymbol{\lambda}}}$, for some choice of $\underline{\underline{\boldsymbol{\lambda}}}$ with $\underline{\underline{\lambda}}_{i} \geq 0$. The basis 

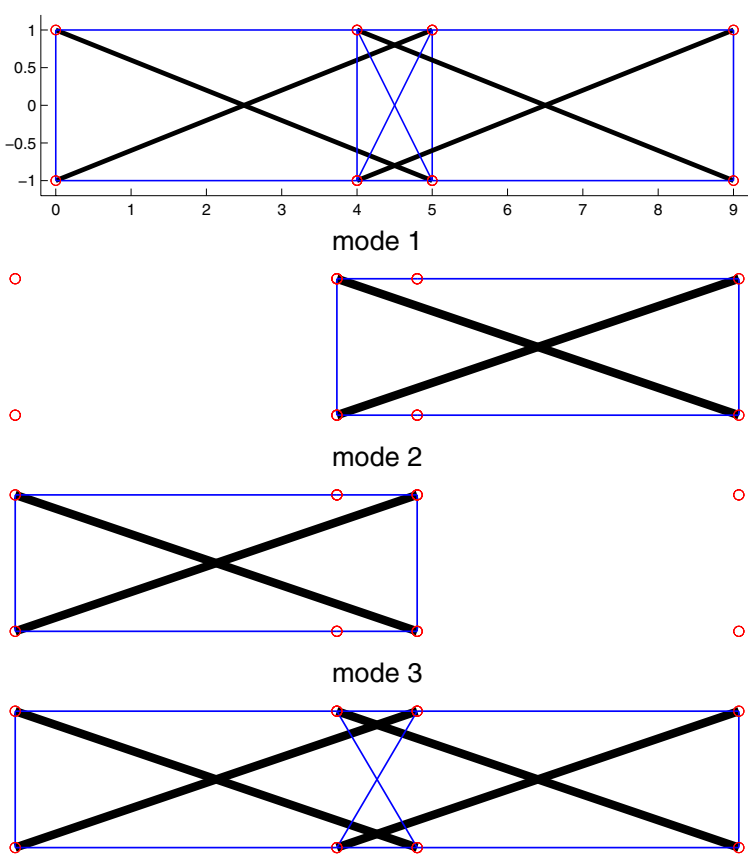

Fig. 1. Tensegrity structure with three prestress modes (top), and the three extreme direction of its prestress cone

for the prestress cone with this property can be computed applying an algorithm that is derived from an implementation of the simplex method for linear programming. This algorithm seeks and identifies all vertices of the linear constraint $N x \geq 0$. Fig. 1 depicts an application of the algorithm on identifying the extreme directions of the prestress cone of the structure that has three prestress modes.

The prestress parameters, $\boldsymbol{\lambda}$, must be additionally constrained to ensure that the actual elements of the structure can be built after defining the prestress of the structure. If the cross-sectional area of the elements, denoted $\mathbf{a}$, is given, and the material of the structure is fixed, by fixing its Young's modulus $\mathbf{y}$, this constraint must guarantee that the resulting element rest-lengths are feasible, that is $l_{0_{i}}>0$. Hence, from (6), force density variables must satisfy,

$$
\lambda_{i} l_{i}<e_{i} a_{i}, \quad e_{i} \in \mathbb{E}_{b} .
$$

Imposing a similar but more restrictive constraint,

$$
\lambda_{i}<\epsilon \frac{e_{i} a_{i}}{l_{i}}, \quad 0<\epsilon<1,
$$

on all elements of the structure ensures that the prestress of the elements does not exceed their yield stress, and constrains the structure from this mode of failure.

\section{THE DESIGN OF THE STRUCTURE FOR THE OPTIMAL LQR PERFORMANCE - OPTIMIZATION OVER THE PRESTRESS CONE}

\section{A. Formulation of the problem}

We assume that only the bar elements are used for controlling a structure resulting in the following reduction of the number of control variables,

$$
\Delta \boldsymbol{\lambda}=\left[\begin{array}{c}
0_{n_{s} \times n_{b}} \\
I_{n_{b}}
\end{array}\right] \Delta \boldsymbol{\lambda}_{b} .
$$

This and the fact that the bars can sustain both compression and tension eliminate the need for imposing additional saturation constraints on the control variables.

The assumption is made that the covariance matrix of external disturbance $W$, and the control and output variance penalty matrices $R \succ 0$ and $Q \succeq 0$ are given. The problem of the optimal selection of structure prestress for the optimal LQR performance can be cast in the following form:

$$
\begin{aligned}
& \min _{\underline{\underline{\boldsymbol{\lambda}}}, G} \operatorname{Tr}\left[\left(G^{T} R G+Q\right) X\right] \\
& \text { subject to } \\
& (A(\underline{\underline{\boldsymbol{\lambda}}})+B G) X+X(A(\underline{\underline{\boldsymbol{\lambda}}})+B G)^{T}+B_{w} W B_{w}^{T}=0, \\
& \underline{\underline{\boldsymbol{\lambda}}} \geq 0, \\
& \lambda_{i} \leq \epsilon \frac{a_{i} y_{i}}{l_{i}}, \\
& \text { where } \quad \boldsymbol{\lambda}=\Lambda \underline{\underline{\boldsymbol{\lambda}}}
\end{aligned}
$$

\section{B. An algorithm for designing the structure for optimal $L Q R$ performance}

The following algorithm provides a monotonic decrease of the cost function of the problem (14) if an initial guess for $\underline{\underline{\lambda}}$ yields a stable matrix $A(\underline{\underline{\boldsymbol{\lambda}}})$.

Step 1. Pick feasible $\underline{\underline{\boldsymbol{\lambda}}}, A(\underline{\underline{\boldsymbol{\lambda}}})$.

Step 2. Solve the LQR proble for $X$ and $G$ ignoring the constraint on feasible $\underline{\underline{\lambda}}$.

Step 3. Compute the gradient grad of $\left(G^{T} R G+Q\right) X$ with respect to $\underline{\underline{\lambda}}$.

Step 4. Compute the projected gradient gradproj with respect to constraint on feasible $\underline{\underline{\lambda}}$.

Step 5. Update $\underline{\underline{\lambda}}$ and $A(\underline{\underline{\lambda}})$ using the line search parameter $\alpha$ that yields a sufficient decrease of the objective, and satisfies that $\underline{\boldsymbol{\lambda}}-\alpha$ gradproj remains feasible.

Step 6. Repeat Steps $2-\overline{5}$ until optimality conditions are satisfied.

1) Computing the gradient of the problem objective function:

Theorem 1: The sensitivity $\partial f\left(x^{*}(y)\right) / \partial y$ of the following optimization problem

$$
\begin{array}{rl}
x^{*}(y)=\arg \min _{x} & f(x) \\
\text { subject to } & c(x, y)=0
\end{array}
$$

is

$$
\frac{\partial f\left(x^{*}(y)\right)}{\partial y}=\left.\frac{\partial L}{\partial y}\right|_{x=x^{*}, t=t^{*}}=\left.t^{* T} \frac{\partial c(x, y)}{\partial y}\right|_{x=x^{*}}
$$

where $t^{* T}$ is the vector of lagrange multipliers at the optimal point $x^{*}$.

Proof: Lagrangian $L$,

$$
L(x, y, t)=f(x)+t^{T} c(x, y),
$$


of the problem must satisfy,

$$
\begin{aligned}
L\left(x^{*}(y), y, t^{*}(y)\right) & =f\left(x^{*}(y)\right)+t^{* T}(y) c\left(x^{*}(y), y\right)= \\
& =f\left(x^{*}(y)\right) .
\end{aligned}
$$

at any optimal point $x^{*}, t^{*}$. Differentiating the Lagrangian at the optimal point, $x^{*}, t^{*}$, yields,

$$
\begin{array}{r}
\frac{\partial f\left(x^{*}(y)\right)}{\partial y}=\frac{\partial L\left(x^{*}(y), y, t^{*}(y)\right)}{\partial y}= \\
=\left.\frac{\partial L}{\partial x}\right|_{x=x^{*}, t=t^{*}} \frac{\partial x^{*}(y)}{\partial y}+\left.\frac{\partial L}{\partial t}\right|_{x=x^{*}, t=t^{*}} \frac{\partial t^{*}(y)}{\partial y}+ \\
\left.\frac{\partial L}{\partial y}\right|_{x=x^{*}, t=t^{*}},
\end{array}
$$

and finally, using the first order optimality conditions, $\left.\frac{\partial L}{\partial x}\right|_{x=x^{*}, t=t^{*}}=0,\left.\frac{\partial L}{\partial t}\right|_{x=x^{*}, t=t^{*}}=0$,

$$
\frac{\partial f\left(x^{*}(y)\right)}{\partial y}=\left.\frac{\partial L}{\partial y}\right|_{x=x^{*}, t=t^{*}}=\left.t^{* T} \frac{\partial c(x, y)}{\partial y}\right|_{x=x^{*}} .
$$

Theorem 1 suggests a method for computing the gradient of the objective function. From the equation in (15), computation of the gradient involves the dual variables $t^{*}$. The dual problem associated with the LQR problem in the Step 2 of the solution algorithm is,

$$
\min _{G} \operatorname{Tr}\left[B_{w} W B_{w}^{T} T\right]
$$

$\underline{\underline{\lambda}}, G$

subject to

$$
(A(\underline{\underline{\boldsymbol{\lambda}}})+B G)^{T} T+T(A(\underline{\underline{\boldsymbol{\lambda}}})+B G)+G^{T} R G+Q=0,
$$

so that, after finishing the execution of the Step 2, the dual variables in $T=T^{T}$ can be recovered by solving the Lyapunov equation,

$$
(A(\underline{\underline{\boldsymbol{\lambda}}})+B G)^{T} T+T(A(\underline{\underline{\boldsymbol{\lambda}}})+B G)+G^{T} R G+Q=0 .
$$

An equivalent form of the Theorem 1 for the problems involving matrices results in the following,

$$
\frac{\partial f\left(G^{*}(\underline{\underline{\boldsymbol{\lambda}}})\right)}{\partial \underline{\underline{\underline{\lambda}}}}=\frac{\partial L^{*}}{\partial \underline{\underline{\underline{\lambda}}}}
$$

where,

$$
\begin{array}{r}
L^{*}=\operatorname{Tr}\left[\left(G^{T} R G+Q\right) X\right]+ \\
\operatorname{Tr}\left[T\left((A(\underline{\underline{\boldsymbol{\lambda}}})+B G) X+X(A(\underline{\underline{\boldsymbol{\lambda}}})+B G)^{T}+B_{w} W B_{w}^{T}\right)\right] .
\end{array}
$$

Using several properties of the trace operator, the gradient of the objective function can be defined as,

$$
\operatorname{grad}_{i}=\frac{\partial L^{*}}{\partial \underline{\underline{\boldsymbol{\lambda}}}_{i}}=2 \operatorname{Tr}\left[\mathbf{M} V_{2}[X T]_{1,2} \underline{\mathcal{M}}^{-1} V_{2}^{T} \mathbf{C} \hat{\Lambda}_{i}\right] .
$$

\section{Example \\ D. Example}

The planar tensegrity structure depicted in Figure 2 is optimized. The structure is supported at the two leftmost nodes. A vertical zero mean white noise disturbance of a unit variance acts at the top rightmost node. The initial distribution of the prestress is uniform, $\underline{\underline{\lambda}}^{T}=\left[\begin{array}{lll}0.1 & 0.1 & 0.1\end{array}\right]^{T}$, as depicted in upper structure of Fig. 2.
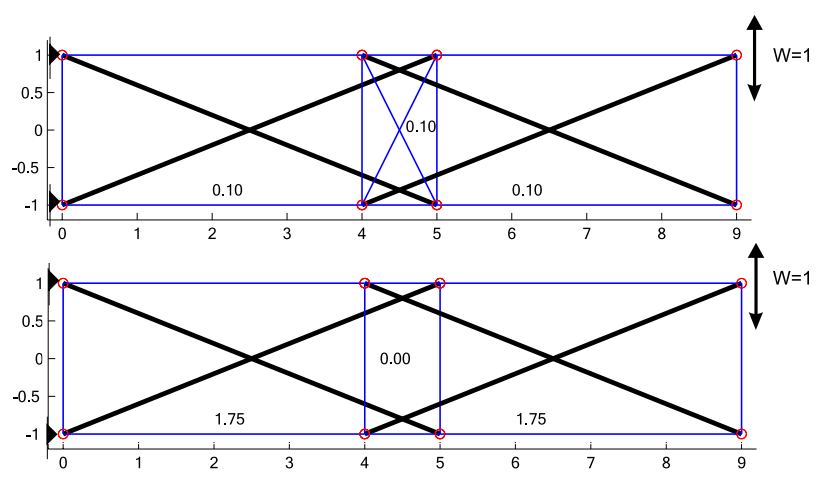

Fig. 2. Initial (up) vs. optimal (down) distribution of prestress in the tensegrity structure

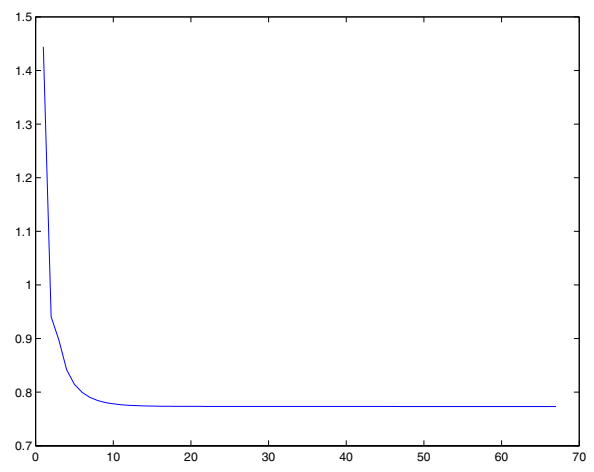

Fig. 3. Convergence of the algorithm-objective vs. iteration number

The outputs of interest are the nodal positions of all free nodes. The optimal distribution of the prestress is not uniform as indicated in the lower structure of Fig. 2. Only the elements that are prestressed are shown. Note that the two diagonal strings in the middle of the structure are slack since the corresponding prestress parameter is zero. The optimal prestress of all four bars is on its upper bound.

The effect of the optimal prestress on the open-loop plant is depicted in Fig. 4. This figure shows the vertical displacement of the top right node, and it is obvious that the optimal distribution of the prestress stiffens the plant. The closed loop behavior of the system is shown in Fig. 5 depicting the vertical displacement of the top right node. It is clear that the $L_{2}$ norm of the plant output is reduced compared to the initial plant for this particular choice of the penalty matrix $R=I$, and the matrix $Q=$ blockdiag $\{I, 0\}$ that penalizes displacements of the free nodes but not their velocities. The same holds true for the control energy, although this is not a general rule independent from the choice of the matrices $Q$ and $R$. Fig. 6 shows the control action of the controlled bar that is attached to the top right node. Clearly, the optimal LQR controller for the optimized plant requires less control energy than the initial optimal controller for the suboptimal plant. 


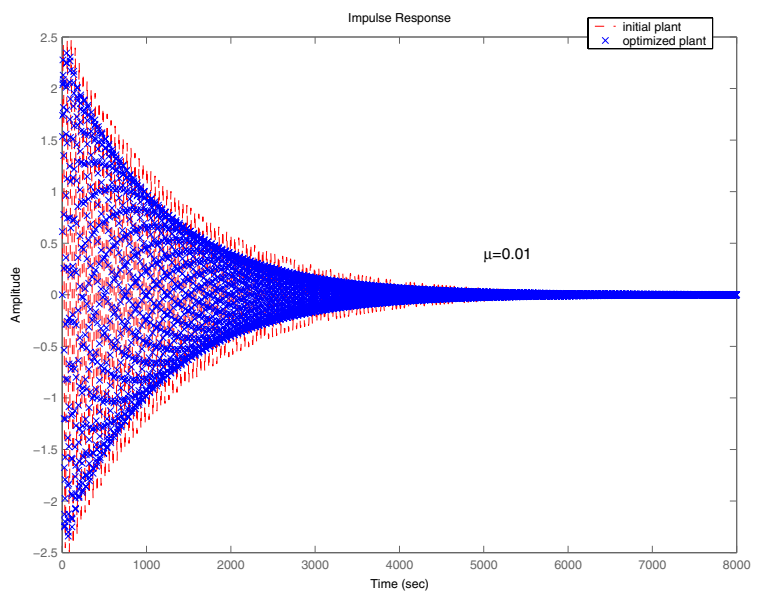

Fig. 4. Impulse response of the initial plant Vs. impulse response of the optimized plant - effect of the structure optimization on its dynamic performance

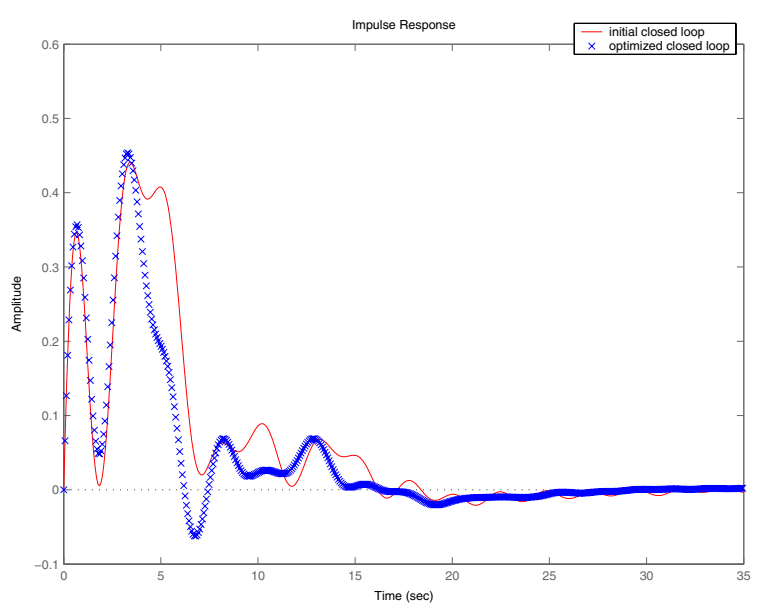

Fig. 5. Closed-loop impulse response of the initial plant Vs. the same response for the optimized plant

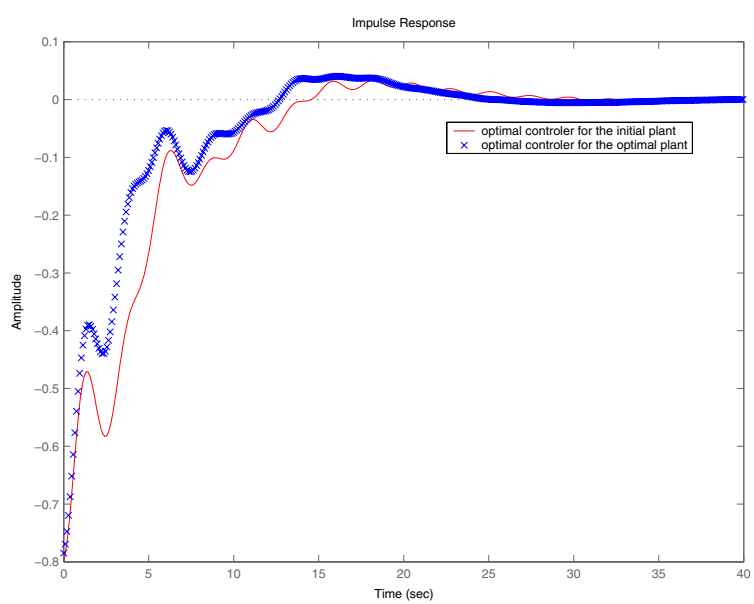

Fig. 6. Output of the controller for the initial and the optimized plant

\section{CONCLUSIONS}

This paper demonstrates a method for integrated plant and control design of tensegrity structures. A gradient based algorithm is proposed for solving the prestress selection problem. The objective gradient was computed analytically using the problem sensitivity analysis. There is no guarantee that the solution shown is a global optimum because of the non-convex nature of the problem. Nonetheless, the algorithm proposed guaranties a monotonic improvement of the performance of the system, and therefore can be considered a legitimate tensegrity design tool. One of the novelties introduced in this paper is the demonstration of the decomposition of the prestress of the structure into the extreme directions of the prestress cone.

\section{REFERENCES}

[1] R. Motro. Tensegrity systems: The state of the art. International Journal of Space Structures, 7(2):75-83, 1992.

[2] A. Hanaor. Double-layer tensegrity grids - static load response. 1. analytical study. Journal of Structural Engineering-ASCE, 117(6):16601674, 1991.

[3] N. Vasart and R. Motro. Multiparametered formfinding method: application to tensegrity systems. International Journal of Space Structures, 14(2):147-154, 1999.

[4] C. Sultan and R. E. Skelton. The prestressability problem of tensegrity structures. some analytical solutions. International Journal of Solids and Structures, 38(30-31):5223-5252, 2001.

[5] M. Masic, R. E. Skelton, and P. E. Gill. Algebraic tensegrity form-finding. International Journal of Solids and Structures, 42(1617):4833-4858, 2005. doi:10.1016/j.ijsolstr.2005.01.014.

[6] M. Masic and R. E. Skelton. Optimization of class-2 tensegrity towers. In Proceedings of the $11^{\text {th }}$ Smart Structures and Materials Conference, volume 5390, pages 163-174, Belligham, WA, USA, 2004. International Society for Optical Engineering (SPIE).

[7] B. de Jager, M. Masic, and R. E. Skelton. Optimal topology and geometry for controllable tensegrity systems. In Proceedings of $15^{\text {th }}$ IFAC World Congress, Barcelona, Spain, 2002.

[8] R. E. Skelton, J.P. Pinaud, and D. L. Mingori. Dynamics of the shellclass of tensegrity structures. Journal of the Franklin Institute, 23(338):255-320, 2001.

[9] H. Murakami. Static and dynamic analysis of tensegrity structures. part 1: Nonlinear equations of motion. International Journal of Solids and Structures, 38(20):3599-3613, 2001.

[10] M. Masic and R. E. Skelton. Path planing and open-loop shape control of modular tensegrity structures. AIAA Journal of Guidance, Control, and Dynamics, 28(3):421-430, May-June 2005.

[11] M. Masic and R. E. Skelton. Open-loop control of class-2 tensegrity towers. In Proceedings of the $11^{\text {th }}$ Smart Structures and Materials Conference, volume 5383, pages 298-308, Belligham, WA, USA, 2004. International Society for Optical Engineering (SPIE).

[12] M. Masic and R. E. Skelton. Open-loop shape control of stable unit tensegrity structures. In Proceedings of the $3^{\text {rd }}$ World Congress of Structural Control, volume 2, pages 439-447, Como, Italy, 2002. John Wiley \& Sons.

[13] C. Sultan and R.E. Skelton. Force and torque smart tensegrity sensor In Proceedings of the $5^{\text {th }}$ Anual Symposium on Smart Structures and Materials, volume 3323, pages 357-368, Belligham, WA, USA, 1998. International Society for Optical Engineering (SPIE).

[14] C. Sultan and R.E. Skelton. Tendon control deployment of tensegrity structures. In Proceedings of the $5^{\text {th }}$ Anual Symposium on Smart Structures and Materials, volume 3323, pages 455-466, Belligham, WA, USA, 1998. International Society for Optical Engineering (SPIE).

[15] C. Sultan, M. Corless, and R. E. Skelton. Symmetric reconfiguration of tensegrity structures. International Journal of Solids and Structures, 39(8):2215-2234, April 2002.

[16] N. Kanchanasaratool and D. Williamson. Modeling and control of class nsp tensegrity structures. International Journal of Control, 75(2):123-139, 2002.

[17] R. E. Skelton. Integrated plant and controller design. American Control Conference, Seattle, June 21-23,, 1995. 
[18] J.B. Lu and R.E. Skelton. Integrating structure and control design to achieve mixed $\mathrm{h}-2 / \mathrm{h}$-infinity performance. International Journal of Control, 73(16):1449-1462, 2000.

[19] R.E. Skelton, B.R. Hanks, and M. Smith. Structure redisign for improved dynamic-response. Journal of Guidance, Control and Dynamics, 15(5):1272-1278, 1992.

[20] Maurício Carvalho de Oliveira, Juan. F. Camino, and Robert E. Skelton. A convexifying algorithm for the design of structured linear controllers. In Proceedings of the 39th CDC Conference, pages 27812786, Sydney, Australia, 2000.

[21] Juan Francisco Camino, Maurício Carvalho de Oliveira, and Robert E. Skelton. 'Convexifying' linear matrix inequality methods for integrating structure and control design. Journal of Structural Engineering ASCE, 129(7):978-988, 2003.

[22] Juan F. Camino, Maurício Carvalho de Oliveira, and Robert E. Skelton. Plant and control design using convexifying LMI methods. In Proceedings of the 15th IFAC Conference, pages 2405-2410, Barcelona, Spain, 2002.

[23] M. Masic. Design, Optimization, and Control of Tensegrity Structures. $\mathrm{PhD}$ thesis, University of California San Diego, Department of MAE, La Jolla, CA, USA, May 2004. 